\title{
Absolute determination of the X-ray absorption coefficient of barium in the $L$ region using a liquid absorption cell
}

\author{
Alojz Kodre, ${ }^{\text {a,b }}$ Jana Padežnik Gomilšek, ${ }^{{ }^{*} *}$ Robert Hauko, ${ }^{c}$ Martin Šala ${ }^{e}$ \\ and Iztok Arčon ${ }^{d, b, f}$
}

A method of absolute measurement of X-ray absorption coefficient is proposed, on the basis of the use of an adjustable pathlength liquid cell and a solution of the investigated element. A variational analysis of the measured data is developed to remove some sources of systematic error. A check of the exponential attenuation with thickness is used to suppress beam harmonics admixture and dark current drifts. The method is illustrated by a measurement on Ba in the $L$ edge region, using aqueous solution of Ba nitrate. Measured data provide information on detailed energy dependence of the absorption beyond the Victoreen-like trends reproduced in compilations. The proposed method provides a way to measure the absorption data for practically all elements and a wide range of photon energies. Copyright @ 2012 John Wiley \& Sons, Ltd.

\section{Introduction}

In X-ray absorption fine structure (XAFS) measurements on modern synchrotron sources, the vicinity of X-ray absorption edges is routinely measured with fine resolution below $1 \mathrm{eV}$ in photon energy and with high precision to detect $\sim 0.1 \%$ changes in intensity of the beam. Although the measurements probe the absorption coefficient of a material, only its relative changes are resolved in the analysis of the material structure. In a relative measurement, some fundamental parameters such as the thickness of the sample and the efficiency of detectors do not enter the analysis and consequently, do not contribute to the systematic error of the results. In an absolute measurement, such parameters need to be carefully determined or eliminated in auxiliary steps of a well-planned experiment.

The strong interest in the measurement of absorption in the pioneering years of X-ray physics waned considerably with the improvement of theoretical methods. Modern compilations of absorption data ${ }^{[1,2]}$ are based mostly on theoretical results and interpolation. The compilations claim a $1 \%$ accuracy in the "smooth « energy regions far from absorption edges and 4\%-20\% in the vicinity of the absorption edges even in monatomic gases free of XAFS modifications of the absorption. Direct measurements have since only been performed on a few selected elements for the purpose of recalibration of compiled data ${ }^{[3,4]}$ or in conjunction with inner-shell atomic spectroscopy. ${ }^{[5]}$ It has been realized that with the advance of X-ray sources and detectors, the accuracy of the absorption coefficient depends mostly on the definition of the sample rather than on the spectroscopic measurement itself. With exception of gaseous absorbers, it is the thickness of the sample that introduces the main error of the result.

For soft X-rays below 5-keV photon energy, the absorption data, both theoretical and experimental, are less reliable, so new measurements and new techniques are well warranted, in particular in the $L$ edge region. ${ }^{[6,7]}$ Below $5 \mathrm{keV}$, the optimum surface density of elemental absorbers is of the order of $1 \mathrm{mg} / \mathrm{cm}^{2}$, which is, at the required precision and homogeneity, hard to achieve by mechanical means and also by evaporation or sputtering techniques. Dilution of the absorber with a low-absorption matrix can be of help, such as in the routine preparation of pellets with admixture of boron nitride in XAFS techniques. A solution of the measured substance in a low- $Z$ liquid provides an ideally homogeneous sample, with the additional advantage of a relatively manageable structural signal.

We demonstrate the absolute measurement of the absorption coefficient of $\mathrm{Ba}$ in the region of $L$ edges (5000-6400 eV) using the solution of Ba nitrate. For the thin and even windows of the sample container, silicon nitride (SiN) membranes have recently become available. They are chemically inert, perfectly flat, and sufficiently transparent in the entire energy range of conventional synchrotron beamlines down to $3 \mathrm{keV}$. Spectrometric liquid cells with reproducibly adjustable thickness in the submillimeter range are also available. The experiment involves several steps: absorption measurements at a sequence of thickness settings of the solution and a separate sequence with the pure solvent

* Correspondence to: Jana Padežnik Gomilšek, Faculty of Mechanical Engineering, University of Maribor, Smetanova 17, SI-2000 Maribor, Slovenia. E-mail: jana.padeznik@uni-mb.si

a Faculty of Mathematics and Physics, University of Ljubljana, Jadranska 19, SI-1000 Ljubljana, Slovenia

b J. Stefan Institute, Jamova 39, SI-1000 Ljubljana, Slovenia

c Faculty of Mechanical Engineering, University of Maribor, Smetanova 17, SI-2000 Maribor, Slovenia

d University of Nova Gorica, Vipavska 13, SI-5000 Nova Gorica, Slovenia

e National Institute of Chemistry, Hajdrihova 19, SI-1000 Ljubljana, Slovenia

f Centre of Excellence Low-Carbon Technologies (CO NOT), Hajdrihova 19, SI-1000 Ljubljana, Slovenia 
or a diluted solution. The rich redundancy of the data is exploited to eliminate some systematic effects in the nonexponential extinction and in the definition of the thickness scale.

\section{Experiment}

By dissolving $0.7938 \mathrm{~g}$ of $\mathrm{Ba}\left(\mathrm{NO}_{3}\right)_{2}$ salt in $12.2073 \mathrm{~g}$ of water, a $C_{\mathrm{Ba}}=0.245 \mathrm{~mol} / \mathrm{l}$ solution of barium nitrate with the density $\rho_{s}=1.05(1) \mathrm{g} / \mathrm{cm}^{3}$ was prepared. The absorption of the solution was measured in a liquid absorption cell adapted from a standard Variable Pathlength Liquid Spectrophotometer Cell (International Crystal Laboratories) by substituting the rock salt windows with lucite supports holding $500-\mathrm{nm}$ thick $(5 \times 5 \mathrm{~mm}) \mathrm{SiN}$ membranes (Norcada Inc.). The vernier of the cell allowed a reproducible setting of the thickness of the solution layer with a micrometer accuracy.

The experiment was performed at the A1 station of the DORIS (DESY Hamburg) storage ring with a Si 111 double-crystal monochromator with resolution of $0.6 \mathrm{eV}$. The third harmonic component (15-19 keV) was suppressed by the use of a flat $\mathrm{Ni}$ mirror (cutoff at $8.3 \mathrm{keV}$ ) and by detuning the monochromator to $50 \%$ of the rocking-curve maximum. The intensity of the beam was measured with three ionization detectors filled, in succession, with $600 \mathrm{mbar} \mathrm{N}_{2}, 160 \mathrm{mbar} \mathrm{Ar}$, and $230 \mathrm{mbar} \mathrm{Ar}+300 \mathrm{mbar}$ of air. The absorption cell was mounted in a narrow air gap in the vacuum beam guide between the first two detectors; their respective outputs $I_{0}$ and $I_{1}$ define the total attenuation length $\mu d=\ln \left(I_{0} / I_{1}\right)$. A $5-\mu \mathrm{m}$ vanadium foil for energy calibration ( $K$ edge $5463.8 \mathrm{eV}$ ) between the last two detectors provided a check on the reproducibility of the monochromator movement. By adjustments of horizontal and vertical exit slits of the monochromator, the beam was carefully positioned in the center of the cell windows, to minimize the error due to a possible nonparallel mounting of the SiN membranes and the resulting deviations from the nominal sample thickness with rotation of the cell micrometer.

A scan on the empty cell, at the start of the experiment, served to determine the absorption in the SiN windows and the air gap. The absorption of the solution was measured in steps of $100 \mu \mathrm{m}$ of thickness setting between 200 and $500 \mu \mathrm{m}$. The interval from 5000 to $6400 \mathrm{eV}$ of photon energy encompassing the $L$ edges of Ba (Fig. 1) was scanned in steps of $5 \mathrm{eV}$. Scans with the same step sequence of slightly acidified $\left(\mathrm{HNO}_{3}\right)$ water were taken at cell settings of 200,400 and $600 \mu \mathrm{m}$. The water was introduced into the cell in situ, in several exchanges of the volume to retain the position of the beam and the setting of the micrometer scale. A trace of Ba remained in the sample, showing minute spikes of edge resonances: not so much from insufficient washing, as it turned out in the analysis, but from adsorption on the windows.

Another scan of the $\mathrm{Ba}\left(\mathrm{NO}_{3}\right)_{2}$ solution at the optimum thickness of $400 \mu \mathrm{m}$ was taken with a denser step sequence, appropriate for EXAFS analysis.

\section{Analysis}

The redundancy in the experimental data is used to recognize and eliminate sources of systematic error and, in the end, to improve the signal-to-noise ratio of the results. Some systematic effects are taken care of by the preparation of the experiment. The homogeneity of a liquid absorption sample is perfect, and the even surface on the nanoscale level is ensured by the production process of $\mathrm{SiN}$ membranes. The disclination of the windows is kept below $1 \%$ by the mounting and its effect in the first order

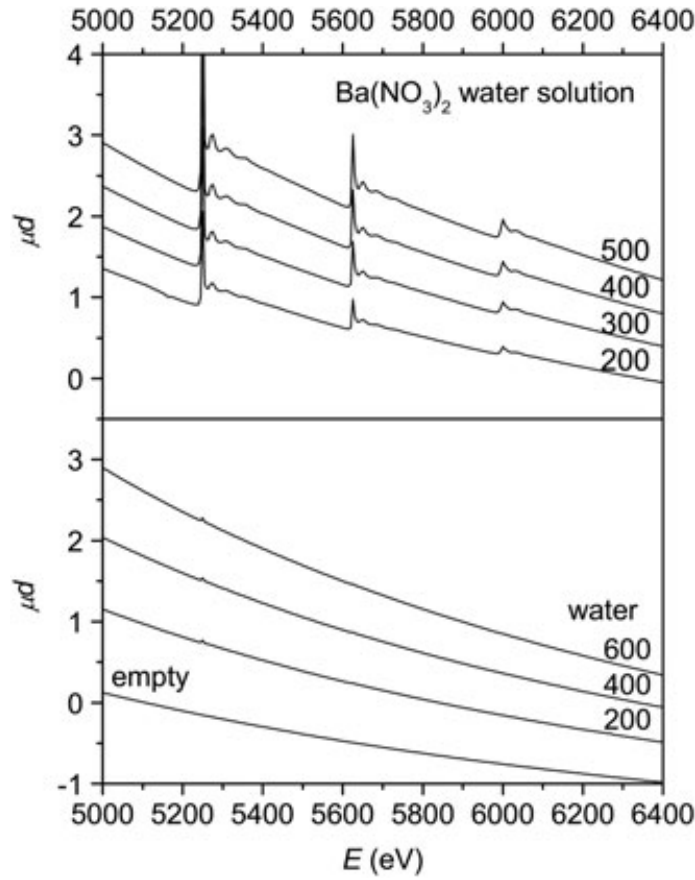

Figure 1. The absorption scans used in the study: a scan on the empty cell, four scans on the $\mathrm{Ba}\left(\mathrm{NO}_{3}\right)_{2}$ solution, and three scans on the water with traces of $\mathrm{Ba}$. Thicknesses of the samples in micrometers are indicated. In the coarse $5 \mathrm{eV}$ step, the edge resonances may not display the proper height.

eliminated by the centering of the beam. Several possible sources of small systematic errors, derived from the beam and detector geometry, are eliminated by using the ratios of the transmitted beam intensities or, equivalently, the differences of attenuation lengths $\mu d$. In this way, systematic errors of the incident beam intensity, common to all thickness settings, are cancelled out. To begin with, the attenuation spectrum of the empty cell as a vector of values at consecutive points of the energy scan is subtracted from the corresponding values in the scan of a liquid sample at the thickness $d_{i}$. In the following discussion, the experimental data prepared in this way will simply be referred to as the absorption spectrum $A_{i}(E)$ at the ith setting of the liquid cell. In the ideal case, the spectra are equal to $\mu(E) d_{i}$, meaning that the ratio $R_{i j}=A_{i}(E) / A_{j}(E)$ should simply be equal to $d_{i} / d_{j}$, a constant over the entire energy range.

The simple picture is modified by possible effects of beam harmonic contamination and of dark current drift of the detectors $^{[4]}$ with the contribution $\varepsilon(E)$ to the transmitted beam: $A_{i}(E)=-\ln \left[\varepsilon(E)+\exp \left(-\mu(E) d_{i}\right)\right]$. Now, the ratios $R$ are not constant: any variation in the ratio, especially any residual of the edge or XAFS features, indicates a contamination. Because third-order harmonics of the beam is thoroughly suppressed in the present experiment, we shall demonstrate the approach for the case of dark current drift that can be modeled by a constant $\varepsilon$. It can be determined by minimization of the variation of the corrected ratio

$$
\underline{R}=\ln \left(-\varepsilon+\exp \left(-A_{\mathrm{i}}\right)\right) / \ln \left(-\varepsilon+\exp \left(-A_{\mathrm{j}}\right)\right)
$$

over the measured energy interval. The process is illustrated in Fig. 2 showing the change of sign of the variations in the ratio $R$ in the vicinity of the optimum value of $\varepsilon$.

The choice of the optimum value of the constant $\varepsilon$ through inspection of the ratio $R$ is not entirely unique: it depends on the judgment when the ratio is best evened out over the spectrum 


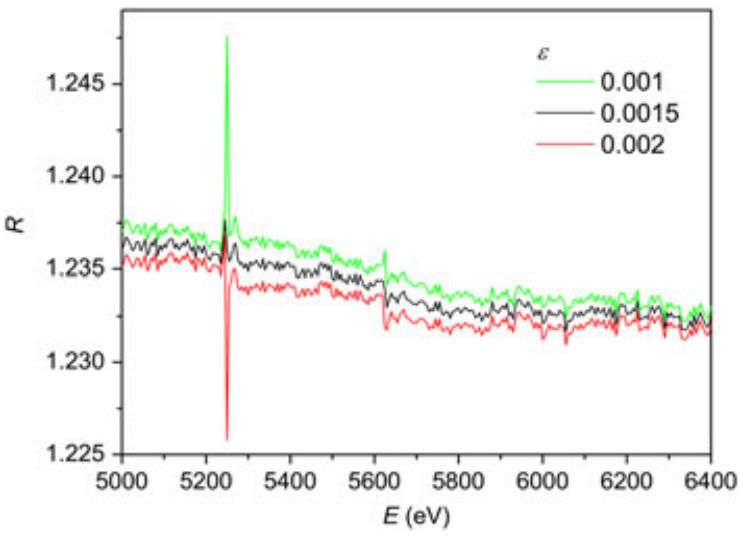

Figure 2. The ratio $R$ of Eqn (1) of spectra at 500 and $400 \mu \mathrm{m}$, at three $\varepsilon$ values close to the optimum. Note that the entire range of $R$ in the graph is $2 \%$, and the effective range outside the resonances $0.3 \%$.

range. In Fig. 2, evidently, the prevailing criterion is the suppression of the edge resonances. The downward trend, although minute, of the ratio is not removed by the choice. The value may also differ for different pairs of spectra in the ratio. A more robust approach is required: a good candidate is based on the least-squares analysis of the data, as explained in the following text.

At a given photon energy $E$, the spectral values $A_{i}(E)$ are expected to depend linearly on the thickness $d_{i}$ (Fig. 3 ), with the slope equal to $\mu(E)$. Small deviations of the measured $A_{i}$ points from the linear relation are strongly correlated for consecutive photon energies: these are the small systematic errors in the micrometer setting persisting through the energy scan. The accuracy of the setting vernier can be estimated to $1 / 10$ of the division, i.e., $1 \mu \mathrm{m}$, so deviations of this size are to be expected.

The linear absorption coefficient $\mu(E)$ of a liquid sample at the energy point $E$ is thus recovered as the best-fit slope of the linear relation between the measured spectral values and the micrometer settings. Let us start with a simple sum of squared deviations

$$
S=\sum_{i}\left[A_{i}(E)-k d_{i}-n\right]^{2}
$$

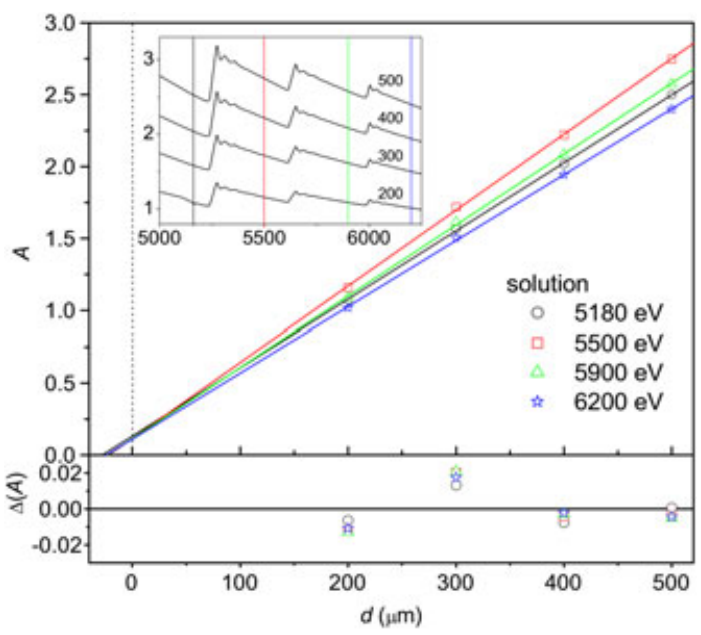

Figure 3. Absorption as a function of micrometer setting at some energy points indicated in the inset. Note the correlated deviations of the experimental values from the respective linear fits shown below on an enlarged scale. in which the parameters $k=\mu(E)$ and $n$ are varied to minimize the sum $S$. The value of $\mu(E)$ is the best average of the values at individual settings, insensitive to the choice of zero of the thickness scale $d_{0}=-n / k$.

The variational approach can be extended to encompass the entire collection of absorption data of a sample, exploiting the redundancy of data along the $E$ coordinate. The minimization of the sum in Eqn (2) can be further restrained by the requirement that the value of $d_{0}$ remain constant along the spectrum. Even more: the correlated deviations of the measured $A_{i}(E)$ from respective straight lines can be interpreted as small errors $\Delta_{\mathrm{i}}$ of the micrometer settings, common to all energy points, and introduced as variation parameters. The absorption coefficient of the solution $\mu_{s}(E)$ is thus obtained by minimization of a grand sum over the energy space

$$
S=\sum_{i} \sum_{j}\left[A_{i}\left(E_{j}\right)-\mu_{s}\left(E_{j}\right)\left(d_{i}+\Delta_{i}-d_{0}\right)\right]^{2}
$$

with additional constraint $\sum_{i} \Delta_{i}=0$. Even the drift correction $\varepsilon$ can be included in the same minimization:

$$
S=\sum_{i} \sum_{j}\left[-\ln \left(-\varepsilon+e^{-A_{i}\left(E_{j}\right)}\right)-\mu_{s}\left(E_{j}\right)\left(d_{i}+\Delta_{i}-d_{0}\right)\right]^{2}(3 a)
$$

In our preliminary analysis, though, the value of $\varepsilon$ was found negligibly small, so that Eqn (3) was used throughout. The value of the zero shift $d_{0}$ was obtained as $(-22 \pm 3) \mu \mathrm{m}$, already indicated in Fig. 3. The shifts $\Delta_{i}$ of consecutive settings are $-2.0,3.5,-1.0$, and $-0.5 \mu \mathrm{m}$, within a $\pm 0.3 \mu \mathrm{m}$ error interval.

The same procedure is applied to the absorption spectra of the solvent whereby the linear absorption coefficient $\mu_{w}(E)$ of pure water is recovered. The vestiges of $\mathrm{Ba}$ edges are suppressed by a factor of $\sim 8$ in the result, confirming that they were mostly due to the constant quantity of the metal adsorbed on the windows. The shift $d_{0}$ is $(-40 \pm 4) \mu \mathrm{m}$, of which almost one half comes from the $\mathrm{Ba}$ on the windows. The $\Delta_{i}$, independent of the previous settings, are $-0.8,1.6$, and $-0.8 \mu \mathrm{m}$. Both linear absorption coefficients are shown in Fig. 4.

The mass absorption coefficient of $\mathrm{Ba}^{2+}$ ion, the proper form of the result for comparison with tabulated absorption data, is calculated by removing the contribution of water and taking into account the density $\rho_{\mathrm{Ba}}=\mathrm{C}_{\mathrm{Ba}} M(\mathrm{Ba})$ of the metal in the solution.

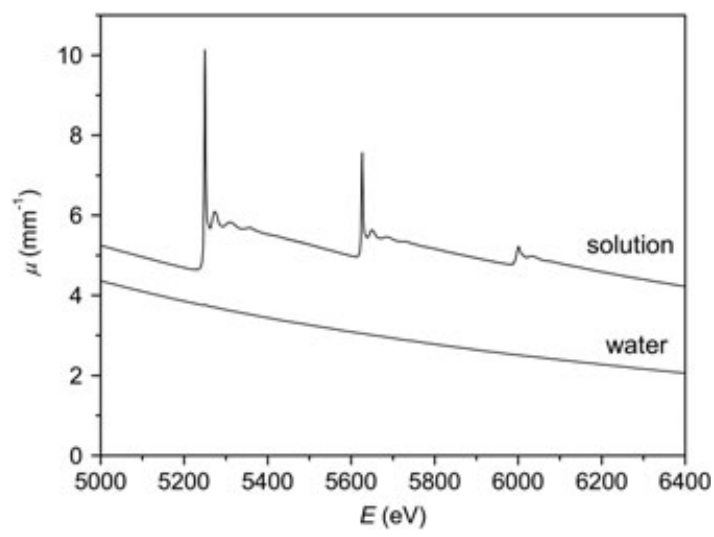

Figure 4. The linear absorption coefficient of the $\mathrm{Ba}\left(\mathrm{NO}_{3}\right)_{2}$ solution and water obtained from variational procedure of Eqn (3); the Ba solution result is projected onto the dense EXAFS scan to reconstruct the full shape of the structural signal and the edge resonances. 


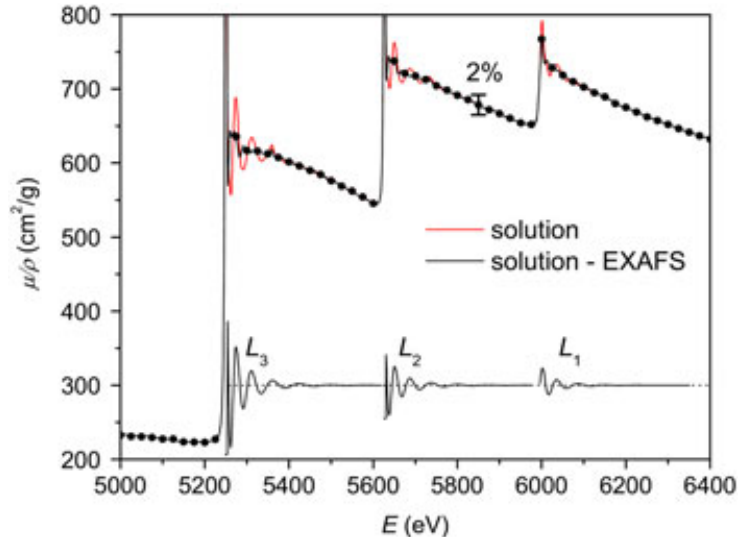

Figure 5. Mass absorption coefficient of $\mathrm{Ba}$ in solution (red) and of atomic Ba (black line and 25-eV step dots). The removed EXAFS signal of the solvation shell (first $\mathrm{O}$ neighbors) is shown below. The error estimate of $2 \%$ is shown.

The contribution of the $\mathrm{NO}_{3}^{-}$anion can be, in view of the small difference between $\mathrm{N}$ and $\mathrm{O}$ absorption, approximated by a fourfold contribution of the same (molar) amount of water:

$$
(\mu / \rho)_{\mathrm{Ba}}=\left[\mu_{\mathrm{s}}-\alpha \mu_{w}\right] / \rho_{\mathrm{Ba}}
$$

where $\alpha=\left[\rho_{s}-c_{\mathrm{Ba}} M\left(\mathrm{Ba}\left(\mathrm{NO}_{3}\right)_{2}\right)+8 c_{\mathrm{Ba}} M\left(\mathrm{H}_{2} \mathrm{O}\right)\right] / \rho_{w}=1.023$, with $\rho_{w}=1 \mathrm{~g} / \mathrm{ml}$.

The accuracy of the spectroscopic measurement itself can be estimated from the ratio $R$ of two independent absorption spectra in Fig. 2 to $0.5 \%$, with statistical noise contributing one third. The overall dispersion of the results obtained from different subsets of the measured spectra is $1 \%$. The contribution of $1 \mu \mathrm{m}$ absolute error of the micrometer settings, reduced by the introduction of variational parameters $\Delta_{i}$, is negligible in comparison.
It turns out, as in most absorption measurements, that an essential source of error of the mass absorption coefficient is the surface density, i.e., the reliability of the chemical definition and preparation of the sample. In our experiment, this is maximized with the estimated $1 \%$ error in the determination of the solution density. With this, the overall relative error of the mass absorption coefficient of $\mathrm{Ba}$ is estimated to $2 \%$.

To use pure water as a reference sample, instead of an equivalent $\mathrm{HNO}_{3}$ solution with the same concentration of the anion whereby Eqn (4) would be somewhat simplified, is preferable in avoiding the measurement of another solution density and thus reducing the error of the side result of water absorption.

\section{Conclusions}

By the transferability principle, ${ }^{[8]}$ the result of the experiment can be used as the basic Ba atom absorption after the details of the edge profiles and XAFS signal, derived from the particular chemical environment, are removed. The oxygen atom signal of the solvation shell using a simple Feff mode $\left.\right|^{[9,10]}$ has been determined from the dense scan of the solution, relying on the detailed analysis in an earlier paper. ${ }^{[11]}$ The removal procedure is illustrated in Fig. 5. A sparse collection of $\mu / \rho$ values is also given in Table 1, together with the values for water after removing Ba residuum.

The measured data provide information on the detailed energy dependence of the absorption that is missing in compilations where only smooth overall trends are reproduced. As in $\mathrm{Xe}, \mathrm{Cs}$, and $\mathrm{Cd}_{1}^{[6,7]}$ the atomic absorption in Ba shows a specific behavior of the absorption coefficient in the interedge regions: it is convex between $L_{3}$ and $L_{2}$ and flat between $L_{2}$ and $L_{1}$. Only above $L_{1}$ it regains the customary concave energy dependence, wellapproximated in wide intervals by the Victoreen power formula. The interedge anomaly is still beyond the reach of self-consistent Dirac-Hartree-Fock calculations used in the modern absorption

Table 1. Mass absorption coefficient of $\mathrm{Ba}$ and water in the region of $L$ edges

\begin{tabular}{|c|c|c|c|c|c|c|c|c|c|c|c|c|c|c|c|}
\hline$E(\mathrm{eV})$ & 5000 & 5100 & 5200 & 5300 & 5400 & 5500 & 5600 & 5700 & 5800 & 5900 & 6000 & 6100 & 6200 & 6300 & 6400 \\
\hline $\mathrm{Ba} \mu / \rho \quad\left(\mathrm{cm}^{2} / \mathrm{g}\right)$ & 233 & 228 & 223 & 617 & 602 & 576 & 545 & 717 & 691 & 667 & & 702 & 675 & 652 & 632 \\
\hline Water $\mu / \rho \quad\left(\mathrm{cm}^{2} / \mathrm{g}\right)$ & 43.6 & 40.9 & 38.5 & 36.3 & 34.3 & 32.5 & 30.8 & 29.2 & 27.7 & 26.3 & 25.0 & 23.8 & 22.7 & 21.6 & 20.5 \\
\hline
\end{tabular}

Relative error is estimated to $2 \%$ and $1 \%$, respectively. The Ba value close to $L_{1}$ edge $(6000 \mathrm{eV})$ is omitted.
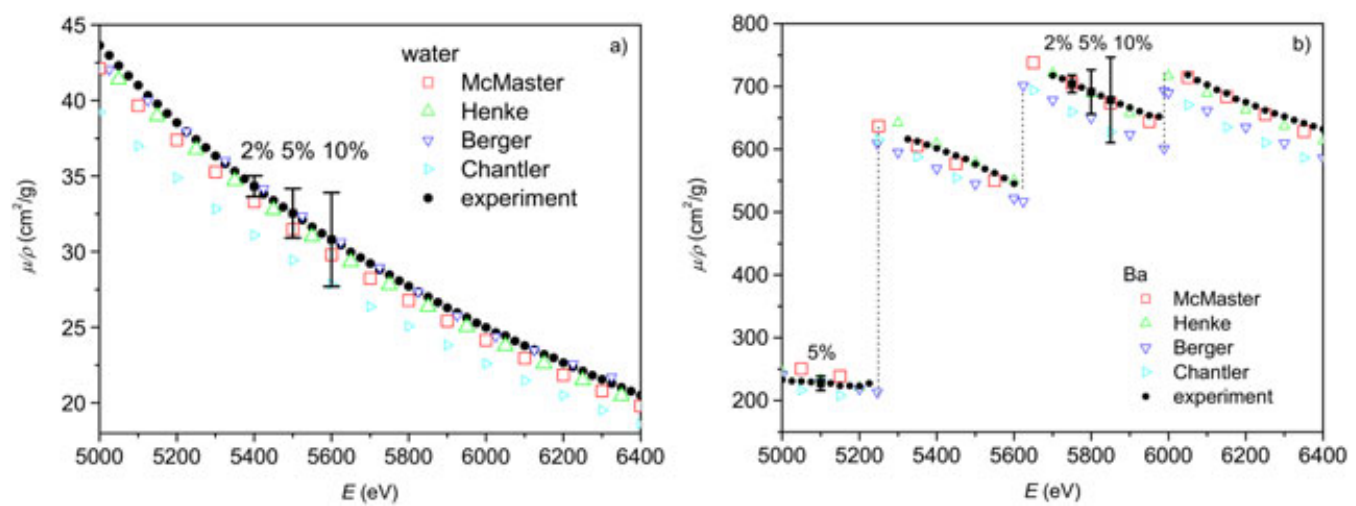

Figure 6. The mass absorption coefficient of water (left) and of Ba (right) in comparison with some tabulated data. The sizes of typical error classes are shown to guide the eye. The points in the near edge regions ( $\sim 50 \mathrm{eV}$ above the edges) are omitted because the midline through the excursions of structural signal cannot be reliably defined. 
tabulations. A close theoretical reconstruction of the profiles, however, using a relativistic random-phase approximation with relaxation has been given for relatively simple case of Xe atom. ${ }^{[12]}$

In Fig. 6, the results of our experiment for $\mathrm{Ba}$ and water are compared with the data from recent $\mathrm{X}$-ray absorption compilations. ${ }^{[1,2,13-15]}$ For these, the density of points required for the comparison has been expanded by the Xafsmass code. ${ }^{[16]}$ The compilation data show good convergence, and they agree with the experiment rather well for Ba below $L_{3}$ edge. Considerable divergence and deviation from experimental data are found in the region between the edges. The earlier tables (Henke and McMaster) with consistently higher values in this region are closer to the experiment. We believe that the procedure employed in our data analysis is particularly effective against the systematic shifts in the absorption coefficient as revealed in the comparison.

The absorption of water is an independent result of our experiment with estimated error of $1 \%$ because the error in the chemical content of the sample is negligible. No earlier measurements in this energy region are reported in Hubbell compilation. ${ }^{[17]}$ The tabulated values deviate from our experiment for $2 \%-10 \%$, with exception of the XCOM (Berger) with 1\% agreement.

We believe that the method presented here provides a way to measure the absorption data for practically all elements and a wide range of photon energies, with a better accuracy than most of the results measured on solid samples.

\section{Acknowledgements}

This work has been supported by the Slovenian Research Agency Research Programme P1-0112, CO NOT Centre of Excellence, and by DESY and the European Community's Seventh Framework Programme (FP7/2007-2013) under grant agreement CALIPSO $n^{\circ}$ 312284 (EU Support of Access to Synchrotrons/FELs in Europe). Access to synchrotron radiation facilities of HASYLAB (project I-20110082 EC) is acknowledged. We would like to thank Roman Chernikov and Edmund Welter of HASYLAB for expert advice on beamline operation.

\section{References}

[1] M. J. Berger, J. H. Hubbell, S. M. Seltzer, J. Chang, J. S. Coursey, R. Sukumar, D. S. Zucker, K. Olsen, XCOM: Photon Cross Sections Database, National Institute of Standards and Technology, Gaithersburg, MD, 1998 [Online]. Available: http://www.nist.gov/pml/data/xcom/ index.cfm [28-Jun-2012].

[2] C. T. Chantler, K. Olsen, R. A. Dragoset, J. Chang, A. R. Kishore, S. A. Kotochigova, D. S. Zucker, X-ray Form Factor, Attenuation, and Scattering Tables, National Institute of Standards and Technology, Gaithersburg, MD, 2001 [Online]. Available: http://www.nist.gov/ $\mathrm{pml} / \mathrm{data} / \mathrm{ffast} / \mathrm{index.cfm} \mathrm{[28-Jun-2012].}$

[3] D. C. Creagh, J. H. Hubbell. Acta Cryst. A 1990, 46, 402.

[4] N. A. Rae, C. T. Chantler, Z. Barnea, M. D. de Jonge, C. Q. Tran, J. R. Hester. Phys. Rev. A 2010, 81, 022904.

[5] A. Kodre, J. Padeznik Gomilsek, A. Mihelic, I. Arcon. Rad. Phys. Chem. 2006, 75, 188.

[6] A. Kodre, J. Padeznik Gomilsek, I. Arcon, G. Aquilanti. Phys. Rev. A 2010, 82, 022513.

[7] J. Padeznik Gomilsek, A. Kodre, I. Arcon, G. Bratina. Phys. Rev. A 2011, $84,052508$.

[8] A. Kodre, R. Preseren, I. Arcon, J. Padeznik Gomilsek, M. Borowski. J. Synchrotron Radiat. 2001, 8, 282.

[9] B. Ravel, M. Newville. J. Synchrotron Radiat. 2005, 12, 537.

[10] J. J. Rehr, R. C. Albers. Rev. Mod. Phys. 2000, 72, 621.

[11] J. Padeznik Gomilsek, A. Kodre, I. Arcon, S. de Panfilis, D. Makovec. J. Synchrotron Radiat. 2011, 18, 557.

[12] M. Kutzner. Radiat. Phys. Chem. 2004, 70, 95.

[13] B. L. Henke, E. M. Gullikson, J. C. Davis. At. Data Nucl. Data Tables 1993, 54, 181.

[14] W. H. McMaster, N. K. Del Grande, J. H. Mallett, J. H. Hubbell, Lawrence Livermore National Laboratory Report UCRL-50174 section II, 1969.

[15] J. H. Hubbell, S. M. Seltzer, Tables of X-ray Mass Attenuation Coefficients and Mass Energy-Absorption Coefficients from $1 \mathrm{keV}$ to $20 \mathrm{MeV}$ for Elements $Z=1$ to 92 and 48 Additional Substances of Dosimetric Interest, National Institute of Standards and Technology, Gaithersburg, MD, 1996 [Online]. Available: http://www.nist.gov/pml/ data/xraycoef/index.cfm [27-Jan-2012].

[16] K. V. Klementiev, XAFSmass, 2012, freeware: www.cells.es/Beamlines/CLAESS/software/xafsmass.html [15-May-2012].

[17] J. H. Hubbell, Bibliography of Photon Total Cross Section (Attenuation Coefficient) Measurements, National Institute of Standards and Technology, Gaithersburg, MD, 1995 [Online]. Available: http://www.nist. gov/pml/data/photon_cs/index.cfm [15-May-2012]. 